Chiron's activity was first noted ${ }^{10}$ when it doubled in brightness in 1988. At the time, it was about $12 \mathrm{AU}$ from the Sun, and the fact that it is active at distances beyond Saturn indicates ${ }^{11}$ that its source of activity is not driven by water-ice sublimation, but by supervolatile ices such as $\mathrm{CO}, \mathrm{CH}_{4}$ or $\mathrm{N}_{2}$. Since 1988, Chiron has displayed almost continuous activity in various outbursts lasting weeks to months with amplitudes ranging from several millimagnitudes to over a full magnitude.

Interestingly, images of Chiron have been found on old astronomical plates, taken before its discovery. Analysis of these images $^{12}$ has shown that Chiron was intrinsically brighter at its last aphelion in 1970 , at 19 AU from the Sun, than in recent years. This distant activity strengthens the case for supervolatiles, but also suggests a complicated variability in the surface activity that may indicate that Chiron's surface is partially or predominantly mantled with inert materials.

The first direct evidence for a coma around Chiron was obtained in chargecoupled-device images ${ }^{2}$ that revealed the presence of small (micrometre-sized) particulates surrounding the object. This particulate coma has since been observed out to distances more than $3 \times 10^{5} \mathrm{~km}$ from Chiron. Evidence for gas in Chiron's coma was obtained ${ }^{13}$ by spectroscopic observations of the efficiently fluorescing tracer species, CN.

Elliot et al. have now extracted fascinating new information about Chiron from photometric studies of the transmission of starlight through Chiron's coma during a rare stellar occultation that occurred in early 1994. The original motivation for observing this occultation was to obtain precise information on Chiron's size and shape, which requires that several stations observe the event. Unfortunately, only two groups, one aboard the NASA Kuiper Airborne Observatory (KAO) and one at the South African Astronomical Observatory (SAAO) obtained data.

With only two lightcurves, a definitive determination of Chiron's precise diameter was not possible. However, something else was found that many will consider much more exciting - the direct detection of opacity structures in the inner few hundred kilometres of Chiron's coma. No such features have ever been reported in any previous stellar occultation by a cometary coma.

Elliot and co-workers' data give clear evidence for no fewer than four discrete 'dust' structures, with optical depths ranging from a few to almost 100 per cent. The number and complexity of the various structures probed in the March 1994 occultation is surprising. And intriguingly, although one of these features ('feature 3') appears to be a broad fan of material, preferentially located in Chiron's orbital plane, features 1,2 and perhaps 4 are indicative of particulate arches or jets, presumably emanating from discrete sites on Chiron's nucleus. If this interpretation is correct (additional occultation data are clearly desired), it confirms recent modelling studies ${ }^{14}$ that suggest that Chiron's activity, and hence its coma, may be generated by a low-gravity analogue of the geyser-like vents that Voyager 2 observed on Neptune's large satellite Triton.

The Triton analogy, if confirmed, would suggest a possible new connection between Kuiper disk objects and their suspected larger relatives, Triton and Pluto. A particularly important question now on the table is whether Chiron's surface activity and atmosphere is generated by the super-volatile $\mathrm{CO}$, which is common in comets, or by $\mathrm{N}_{2}$, which is the dominant volatile on Pluto and Triton. If this chemical question can be answered, we will have gained a telling piece of evidence to place the Kuiper disk objects now being discovered in context between the comets and Pluto and Triton.

The observations made by Elliot et al. have added impetus to studies of Chiron at a particularly relevant time: Chiron is just now approaching its perihelion, which will be reached in February 1996. Chiron's approach to and passage through perihelion will offer a valuable opportunity to study this fascinating object at four times closer range than its cohorts in the Kuiper disk. The combination of this difference in distance and Chiron's activity makes Chiron appear more than 250 times brighter than $\mathrm{QB}_{1}$. Perihelion also offers the chance to see this putative Kuiper disk refugee subjected to over an order of magnitude more heating than objects undergo in the Kuiper disk, thereby allowing astronomers to probe its composition, study its surface physics and observe its coma with unprecedented clarity.

Chiron's last perihelion passage occurred in 1945, long before its discovery. The growing body of evidence about this object indicates astronomers will not pass up this opportunity for its successor, in 2047.

S. Alan Stern is in the Southwest Research Institute, Boulder, Colorado 80302, USA.

1. Elliot, J. L. et al. Nature 373, 46-49 (1995)

2. Meech, K. J. \& Belton. M. J. S. Astr. J. 100, 1323 (1990).

3. Boice, D. C. et al in Proc. Distant Comet Activity Workshop (eds Keller, H. U. \& Huebner, W.) 134-139 (Southwest Research Institute, San Antonio, 1993).

4. Kuiper, G. P. in Astrophysics: A Topical Symposium (ed. Hynek, J. A.) 357 (McGraw Hiil, New York, 1951).

5. Jewitt, D. \& LuU, J. Nature 362, 730 (1993)

6. Sykes, M. V. \& Walker, R. G. Science 251. 777 (1991).

7. Campins, H. et al. Astr. I. (in the press).

8. Kowal. C. T. in Asteroids (ed. Gohrels. T.) 436-441 (Univ. Arizona Press, 1979)

9. Dones, L. et al. Bull. Am. astr. Soc. 26. 1154 (1994).

10. Tholen, D. J. et al. IAU Circ. no. 4554 (1988).

11. Stern, S. A. Publs astr. Soc. Pacif. 101, 126 (1989).

12. Bus, S. J. Science 251, 774 (1991)

13. Bus, S. J et al. Proc. Distant Comet Activity Workshop, (eds Keller, H. U. \& Huebner, W.) 41-43 (Southwest Research Institute, San Antonio, 1993).

14. Stern, S. A. et al. Astr. J. 107, 765 (1994).

\section{Sight unseen}

There was a young man who said 'God

Must think it exceedingly odd

If he finds that this tree

Continues to be

When there's no one about in the Quad.'

Reply:

Dear Sir, Your astonishment's odd:

I am always about in the Quad.

And therefore the tree

Will continue to be,

Since observed by,

Yours faithfully, GOD.

THESE limericks, at least one of which is by Ronald Knox, discuss the old philosophical notion that objects only exist while you are looking at them. Daedalus now has a video system that works this way. It exploits the fact that only a tiny fraction of our field of view (equivalent to a thumbnail at arm's length) can be seen sharply. Our illusion of wide clear sight is produced by scanning this region of high acuity rapidly around the scene. The process can be followed by bouncing an infrared beam off the eye to detect its changing direction of gaze.

So Daedalus's new video system uses an infrared scanner above the screen to detect the viewer and the direction of his gaze. When no one is looking at the screen, it displays only a rough, blurred image, of the sort conveyed by our peripheral vision. But as soon as a viewer glances at any point on the screen, the scanner detects the fact, and the image at that point is drawn in full detail. No matter how fast the viewer looks round the image, the electronics is faster. Wherever he glances, he will find the image clear and highly resolved. He will have the illusion of a complete and perfect picture.

The prime application, of course, is high-definition interactive cable television. Each set will carry an infrared scanner sending the direction of its viewer's glance back to the transmitter. Instead of giving him the whole big picture, it need only send the bit he is currently looking at. A tiny bandwidth could relay the illusion of a stunningly detailed image. Two viewers could even watch different channels on the same screen: each would receive mosaic fragments from his own programme.

Virtual-reality computing will be greatly enhanced as well. Working in real time, even the fastest computer can only create a crude, jerky cartoon world for its user to play in or interact with. But with infrared scanner feedback, it need only define explicitly the small chunk he is currently scrutinizing; it can sketch the rest. The resulting richly delineated fantasy world would be doubly illusory.

David Jones 